\title{
Contribuições de testes de tempo de reação para avaliações do comportamento motor e da saúde: software E-Prime ${ }^{\circledR}$
}

\author{
Reaction time software contributions for assessments of motor behavior \\ and health: E-Prime ${ }^{\circledR}$ software
}

Tânia Brusque Crocetta ${ }^{1}$, Alexandro Andrade ${ }^{1}$

${ }^{1}$ Centro de Ciências da Saúde e do Esporte da Universidade do Estado de Santa Catarina (UDESC) - Florianópolis (SC), Brasil.

DOI: http://dx.doi.org/10.7322/abcshs.v39i2.628

\section{RESUMO}

Este estudo analisou a produção científica relacionada ao uso de softwares de tempo de reação (TR), identificando o mais utilizado e descrevendo a temática dos estudos que fizeram uso deste software, a partir de artigos publicados na base de dados ScienceDirect (de 2001 até 2013), por ser abrangente e cobrir as principais áreas de interesse no uso deste tipo de software. A partir das palavras "reaction time" e "computer ou software", identificou-se o software E-Prime ${ }^{\circledR}$ como o mais utilizado, totalizando 11 artigos que foram selecionados para esta pesquisa. Os itens investigados foram: periódico, objetivo do estudo, amostra, área de aplicação ou especialidade, acessório utilizado para resposta, tipo de TR, descrição da tarefa e resultados. O periódico Appetite publicou dois artigos, as demais publicações ocorreram em periódicos distintos. Predominaram pesquisas com adultos saudáveis $(n=5)$, com média de 57,6 participantes. Para registro da resposta, foi utilizado um acessório externo que acompanha o software. Quanto ao tema, foram identificados e apresentados resultados sobre análises da coordenação e planos motores $(n=2)$, análises de transtornos relacionados ao uso de substâncias $(n=2)$, transtornos da visão $(n=1)$, análises no comportamento alimentar $(n=2)$, o efeito da motivação sobre o desempenho no TR $(n=1)$ e os transtornos da fala e da linguagem $(n=2)$, além da avaliação dos distúrbios da atenção em Parkinsonianos $(n=1)$. Os resultados demonstram a diversidade de aplicabilidade de estudos com tarefas de TR com o uso de apenas um software e a forte relação entre TR e medidas de saúde física e cognitiva.

Palavras-chave: tempo de reação; software; transtornos das habilidades motoras.

\section{ABSTRACT}

The study analyzed scientific production related to the use of reaction time software (RT), to identify the most used and describe the main issues of studies that have made use of this software published from 2001 to 2013 in the ScienceDirect electronic database, since this database is comprehensive and covers the main areas of interest with the use of this software. From the words "reaction time" and "computer or software", the E-Prime ${ }^{\circledR}$ software was identified as the most used, totaling 11 articles, which were selected for the study. The items investigated were: journal, objective, sample, application area or specialty, accessory used to reply, RT type, task description and results. The Appetite journal published two articles; the other articles were published in different journals, with predominance of research with healthy adults $(n=5)$ with 57.6 participants on average. To record response in the data collection, it was used an external accessory accompanying the software. In relation to the topic, the results of articles on the analyses of coordination and motor plans $(n=2)$, analyses of disorders related to the use of substance $(n=2)$, vision disorders $(n=1)$, analyses of feeding behavior $(n=2)$, effect of motivation on performance in RT $(n=1)$, speech and language disorders $(n=2)$, and evaluation of attention disorders in Parkinson's patients $(n=1)$ were identified and presented. The results demonstrate the applicability diversity of studies with RT tasks using only one software and the strong relationship between RT and measures of physical and cognitive health.

Keywords: reaction time; software; motor skills disorders. 


\section{INTRODUÇÃO}

Testes de tempo de reação (TR) medem o tempo decorrido entre o aparecimento de um estímulo e a resposta do indivíduo a esse estímulo ${ }^{1-3}$.

Em 2006, Stahl ${ }^{4}$ propôs uma revisão que comparou quatro pacotes de software para geração e execução de experiências psicológicas com uso do computador, dentre elas o tempo de reação: DirectRT, E-Prime, Inquisit e SuperLab. Para cada software, o autor fez uma descrição, considerando a instalação, as características dos estímulos e respostas, uma abordagem geral para a criação de um experimento, o custo e os dados gerados.

O reconhecimento da utilidade da administração de avaliações clínicas em computador coincidiu com os recentes avanços da tecnologia e de computadores pessoais e a produção de numerosas baterias de testes computadorizados ${ }^{5}$.

Em comparação com os tradicionais instrumentos de avaliação neuropsicológica, testes computadorizados também podem representar uma economia de custos potenciais, não só no que diz respeito aos materiais e suprimentos mas também no tempo necessário para administração do teste ${ }^{2,5}$.

A realização de testes de avaliação cognitiva ao longo da vida diária oferece oportunidades para detectar precocemente alterações na eficiência cognitiva, pois permite a identificação dos primeiros sintomas de comprometimento cognitivo em grupos de risco, como os idosos, ou para reduzir o risco de sobrecarga cognitiva para os trabalhadores que realizam tarefas complexas como assistentes de $\mathrm{voo}^{2}$, sendo o TR um teste simples e sensível para a detecção de variação na eficiência cognitiva ${ }^{1,6}$.

Considerando que vários testes com tarefas de TR foram desenvolvidos e que os usuários devem responder a estímulos visuais usando mouse, teclado ou botões especiais ${ }^{1}$ e considerando também a importância do uso de testes com tarefas de TR, o objetivo deste estudo foi analisar a produção científica relacionada ao uso de softwares com tarefas de TR nas diversas áreas, para identificar o software comercial mais utilizado e descrever os principais temas de estudos, a partir de artigos publicados nos anos de 2001 até 2013.

\section{MÉTODO}

Trata-se de uma revisão sistemática, cujo método seguiu as recomendações constantes do Preferred Reporting Items for Systematic Reviews and Meta-Analyses (PRISMA) ${ }^{7}$, uma forma de pesquisa que utiliza como fonte de dados a literatura sobre determinado tema, disponibilizando um resumo das evidências relacionadas a um tema específico, pela utilização de métodos explícitos e sistematizados de busca, apreciação crítica e síntese da informação selecionada ${ }^{8}$.

\section{Estratégia de busca}

A busca pelos artigos foi realizada no mês de dezembro de 2013 na base de dados ScienceDirect ${ }^{\mathbb{B}}$. Analisou-se a produção científica relacionada ao uso de software de TR para mensuração de tarefas motoras a partir de artigos publicados nos anos de 2001 a 2013. A partir das combinações das palavras "reaction time e computer" e "reaction time e software" nos campos "Abstract, Title, Keywords", 121 artigos foram selecionados para a identificação do software utilizado. Após contagem, identificou-se que os dois softwares comerciais mais utilizados foram o E-Prime $^{\circledR}$ (Psychology Software Tools, Inc., Pittsburgh, PA, EUA), com 11 estudos e, em seguida, o Presentation (Neurobehavioural Systems; Albany, CA, EUA), com nove estudos. Os 11 artigos que utilizaram o E-Prime ${ }^{\circledR}$ foram selecionados para análise do texto completo para compor este trabalho (Figura 1).

A busca na base de dados ScienceDirect foi motivada, além de sua larga abrangência, por cobrir as áreas de Ciências da Saúde, Ciências Biológicas, Ciências Agrárias, Ciências Exatas e da Terra, Engenharias, Ciências Sociais Aplicadas, Ciências Humanas e Letras e Artes, com disponibilidade das publicações da Elsevier e de outras editoras científicas, com os textos, em sua maioria, completos. O texto completo foi essencial para esta pesquisa, pois os detalhes do software e das tarefas de TR normalmente não são incluídos nos resumos.

Após seleção dos artigos $(\mathrm{n}=11)$, todos foram lidos na íntegra por dois pesquisadores. $\mathrm{O}$ texto completo foi criteriosamente analisado para compor uma tabela de resumo contendo: autor do estudo, ano da publicação, periódico em que foi publicado, objetivo, amostra, área de aplicação do software e/ou especialidade, tipo de acessório utilizado para indicação da resposta, tipo de TR, descrição da tarefa de TR e principais resultados.

A análise dos artigos selecionados por meio dos itens ou categorias produziu resultados que foram descritos em uma tabela geral, e a discussão foi conduzida nos subitens "O efeito da motivação sobre o desempenho no TR"; "Transtornos relacionados ao uso de substâncias e TR”; “TR na identificação de tendências no comportamento alimentar"; "TR e transtornos da visão”; “TR e comportamento motor" e "TR para avaliação de transtornos da linguagem".

\section{RESULTADOS}

A partir das combinações de palavras "reaction time" E "computer OU software", foram lidos 121 artigos na base de dados pesquisada. A identificação dos softwares utilizados nos estudos resultou na Tabela 1. Após a identificação do E-Prime ${ }^{\circledR}$ como software mais utilizado, 11 artigos compuseram a amostra analisada. Os artigos incluídos na pesquisa são apresentados na Tabela 2, destacando os seguintes dados: autor e ano de publicação, periódico em que foi publicado, objetivo, amostra, área de aplicação do software e/ou especialidade, acessório utilizado para registrar a resposta, tipo de TR, descrição da tarefa de TR e principais resultados.

A primeira publicação registrada nos periódicos investigados ocorreu no ano de 2002, havendo um aumento de artigos que utilizaram o software E-Prime ${ }^{\circledR}$ a partir de 2011. O periódico Appetite 

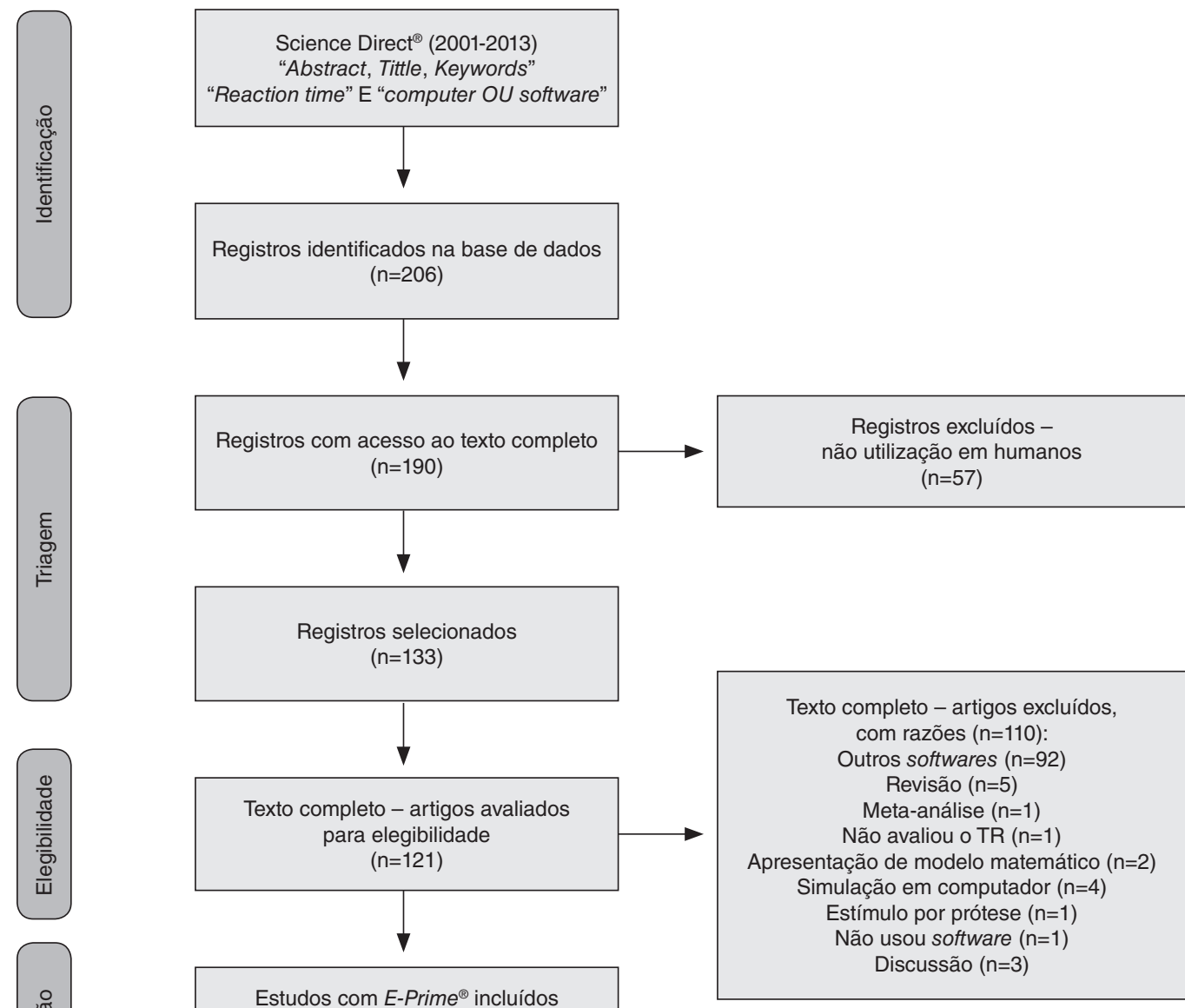

na revisão sistemática $(n=11)$

TR: tempo de reação

Figura 1: Seleção de estudos relacionados ao uso do software E-Prime ${ }^{\circledR}$ em tarefas com tempo de reação publicados na base de dados ScienceDirect nos anos de 2001 a 2013 (adaptado de Moher ${ }^{39}$ )

Tabela 1: Resultado da busca dos softwares de tempo de reação utilizados nos artigos publicados em periódicos indexados na base de dados ScienceDirect nos anos de 2001 a 2013

\begin{tabular}{|l|c|c|}
\hline Software & \multicolumn{1}{c|}{$\begin{array}{c}\text { Fúmero } \\
\text { estudos }\end{array}$} \\
\hline Não mencionou & - & 26 \\
\hline Desenvolvido para a pesquisa & Psychology Software Tools, Inc., Pittsburgh, PA - http://www.pstnet.com/index.cfm & 11 \\
\hline E-Prime ${ }^{\circledR}$ & Neurobehavioural Systems; Albany, CA, USA - http://www.neurobs.com/ & 9 \\
\hline Presentation & Nova Vision AG, Magdeburg & 2 \\
\hline High-Resolution Perimetry (HRP) & Millisecond - http://www.millisecond.com/ & 2 \\
\hline Inquisit & The Psychology Company - http://www.fepsy.com/ & 2 \\
\hline FePsy - The Iron Psyche & National Instruments Corporation - http://www.ni.com/labview/ \\
\hline LabView & Fabricante/distribuidor não localizado & 2 \\
\hline NES2 - Neurobehavioral Evaluation System 2 & Fabricante/distribuidor não localizado & 2 \\
\hline Vscope Software & & 2 \\
\hline Softwares distintos com apenas uma ocorrência & & 2 \\
\hline
\end{tabular}


Tabela 2: Análise dos artigos publicados em periódicos indexados na base de dados ScienceDirect, sobre a temática "software de tempo de reação mais utilizado" que resultou na seleção do E-Prime ${ }^{\circledR}$

\begin{tabular}{|c|c|c|c|c|c|c|c|}
\hline Estudo & Periódico & Objetivo & Amostra & $\begin{array}{c}\text { Área de } \\
\text { aplicação e/ou } \\
\text { especialização }\end{array}$ & AR & $\begin{array}{l}\text { Tipo de TR e } \\
\text { descrição da } \\
\text { tarefa de TR }\end{array}$ & Resultados \\
\hline $\begin{array}{l}\text { Dujardin } \\
\text { et al. } \\
(2013)^{18}\end{array}$ & $\begin{array}{l}\text { Parkinsonism } \\
\text { and Related } \\
\text { Disorders }\end{array}$ & $\begin{array}{l}\text { Investigar a natureza } \\
\text { dos distúrbios de } \\
\text { atenção em pacientes } \\
\text { com doença de } \\
\text { Parkinson }\end{array}$ & $\begin{array}{c}80 \text { pacientes } \\
\text { com doença de } \\
\text { Parkinson, sendo } \\
40 \text { homens (média } \\
\text { idade } 60,93 \pm 9,17 \text { ), } \\
\text { e } 60 \text { adultos } \\
\text { saudáveis (média } \\
\text { idade } 60,53 \pm 9,39 \text { ) } \\
\text { no grupo controle, } \\
\text { sendo } 31 \text { homens. }\end{array}$ & Psicologia & $\begin{array}{l}\text { Acessório } \\
\text { externo }\end{array}$ & $\begin{array}{l}\text { TR = Pressionar a tecla } \\
\text { de resposta o mais } \\
\text { rápido possível quando } \\
\text { um quadrado cinza } \\
\text { apresentado no centro do } \\
\text { monitor alterava para a cor } \\
\text { verde, azul ou vermelha. } \\
\text { TRe = Pressionar a tecla } \\
\text { de resposta o mais rápido } \\
\text { possível quando dois } \\
\text { quadrados eram mostrados } \\
\text { no centro do monitor, } \\
\text { independente da cor. }\end{array}$ & $\begin{array}{l}\text { Resultados evidenciaram } \\
\text { os déficits nos } \\
\text { componentes executivos } \\
\text { da atenção na doença } \\
\text { de Parkinson associada } \\
\text { à baixa capacidade de } \\
\text { focar a atenção, resistir } \\
\text { à interferência e dividir } \\
\text { recursos de atenção. }\end{array}$ \\
\hline $\begin{array}{l}\text { Forestell } \\
\text { et al. } \\
(2013)^{11}\end{array}$ & Appetite & $\begin{array}{l}\text { Determinar se mulheres } \\
\text { que comem de forma } \\
\text { contida ou desenfreada } \\
\text { demonstraram níveis de } \\
\text { atenção diferenciados } \\
\text { para alimentos de alto } \\
\text { teor calórico. }\end{array}$ & $\begin{array}{l}139 \text { mulheres, } \\
\text { estudantes (média } \\
\text { idade } 19,1 \pm 0,4 \text { ), } \\
\text { divididas em dois } \\
\text { grupos: comem } \\
\text { desenfreadamente } \\
\text { ou de forma } \\
\text { contida. }\end{array}$ & $\begin{array}{l}\text { Controle } \\
\text { do apetite } \\
\text { (Segurança } \\
\text { Alimentar e } \\
\text { Nutricional) }\end{array}$ & Teclado & $\begin{array}{l}\text { TRe = Pressionar uma } \\
\text { das teclas do teclado o } \\
\text { mais rapidamente possível } \\
\text { à imagem do alimento } \\
\text { mostrado no monitor, para } \\
\text { responder se gostariam de } \\
\text { comê-lo no café da manhã. }\end{array}$ & $\begin{array}{l}\text { Forneceram evidências } \\
\text { iniciais de que os alimentos } \\
\text { de alto teor calórico } \\
\text { induziram um conflito } \\
\text { de resposta de forma } \\
\text { diferenciada em mulheres } \\
\text { que comem de forma } \\
\text { contida daquelas sem } \\
\text { restrições. }\end{array}$ \\
\hline $\begin{array}{l}\text { Johnson; } \\
\text { Conture; } \\
\text { Walden } \\
(2012)^{30}\end{array}$ & $\begin{array}{l}\text { Journal of } \\
\text { Communication } \\
\text { Disorders }\end{array}$ & $\begin{array}{c}\text { Avaliar os processos } \\
\text { de atenção de gagos } \\
\text { e não gagos em idade } \\
\text { pré-escolar. }\end{array}$ & $\begin{array}{l}24 \text { meninos, sendo } \\
12 \text { gagos ( } 3 \text { a } 5 \\
\text { anos de idade). }\end{array}$ & $\begin{array}{l}\text { Neurolinguagem } \\
\text { (Psicologia } \\
\text { Cognitiva e da } \\
\text { Linguagem) }\end{array}$ & $\begin{array}{l}\text { Acessório } \\
\text { externo }\end{array}$ & $\begin{array}{c}\text { TRe = Tempo decorrido } \\
\text { entre o aparecimento } \\
\text { do estímulo e o } \\
\text { pressionamento do botão } \\
\text { de resposta. }\end{array}$ & $\begin{array}{l}\text { Meninos gagos em idade } \\
\text { pré-escolar apresentam } \\
\text { mesma capacidade de } \\
\text { regular sua atenção do que } \\
\text { os não gagos. }\end{array}$ \\
\hline $\begin{array}{l}\text { van Holst } \\
\text { et al. } \\
(2012)^{12}\end{array}$ & $\begin{array}{l}\text { Journal of } \\
\text { Adolescent } \\
\text { Health }\end{array}$ & $\begin{array}{l}\text { Examinar se as } \\
\text { tendências comumente } \\
\text { relacionadas com } \\
\text { comportamentos } \\
\text { problemáticos de } \\
\text { dependência também } \\
\text { estão relacionadas } \\
\text { ao computador e } \\
\text { videogames em } \\
\text { adolescentes. }\end{array}$ & $\begin{array}{l}92 \text { adolescentes } \\
\text { do sexo masculino } \\
\text { (média idade } \\
15,1 \pm 1,27 \text { ) que } \\
\text { dedicam a maior } \\
\text { parte do seu } \\
\text { tempo em jogos } \\
\text { de computador ou } \\
\text { videogame. }\end{array}$ & $\begin{array}{l}\text { Comportamento } \\
\text { humano }\end{array}$ & Teclado & $\begin{array}{c}\text { TRe = Responder o } \\
\text { mais rápido possível } \\
\text { ao aparecimento de um } \\
\text { quadro em um dos locais } \\
\text { de duas figuras que } \\
\text { desapareciam (sendo uma } \\
\text { figura relacionada com } \\
\text { temas de jogos e a outra } \\
\text { neutra). }\end{array}$ & $\begin{array}{l}\text { Os resultados sugerem } \\
\text { que a indicação de um viés } \\
\text { de atenção em jogadores } \\
\text { de computador ou } \\
\text { videogame é semelhante } \\
\text { a dos dependentes de } \\
\text { substâncias em relação } \\
\text { aos mecanismos cognitivos } \\
\text { emocionais. }\end{array}$ \\
\hline $\begin{array}{l}\text { Eckner; } \\
\text { Chandran; } \\
\text { Richardson } \\
(2011)^{10}\end{array}$ & $\begin{array}{l}\text { Physical } \\
\text { Medicine and } \\
\text { Rehabilitation }\end{array}$ & $\begin{array}{c}\text { Investigar a influência } \\
\text { do feedback e } \\
\text { da motivação no } \\
\text { desempenho durante } \\
\text { dois testes de tempo } \\
\text { de reação simples } \\
\text { visuomotora. }\end{array}$ & $\begin{array}{l}31 \text { adultos } \\
\text { saudáveis (média } \\
\text { idade } 54 \pm 15 \text { ) } \\
\text { que aguardavam } \\
\text { pacientes na sala } \\
\text { de espera de } \\
\text { uma clínica de } \\
\text { reabilitação. }\end{array}$ & $\begin{array}{c}\text { Comportamento } \\
\text { humano }\end{array}$ & Teclado & $\begin{array}{l}\text { TR = Tempo decorrido } \\
\text { entre o aparecimento } \\
\text { de um "X" preto no lugar } \\
\text { de um círculo preto e o } \\
\text { pressionamento da barra } \\
\text { de espaço do teclado. }\end{array}$ & $\begin{array}{l}\text { Participantes motivados } \\
\text { são mais propensos } \\
\text { a apresentar um nível } \\
\text { elevado de esforço durante } \\
\text { a tarefa de TR do que os } \\
\text { participantes desmotivados } \\
\text { ou entediados. }\end{array}$ \\
\hline $\begin{array}{l}\text { Makris; } \\
\text { Hadar; } \\
\text { Yarrow } \\
(2011)^{14}\end{array}$ & $\begin{array}{l}\text { Brain and } \\
\text { Cognition }\end{array}$ & $\begin{array}{l}\text { Explorar a evolução } \\
\text { temporal de planos } \\
\text { motores oferecida por } \\
\text { objetos comuns, para } \\
\text { verificar a teoria de } \\
\text { affordances que implica } \\
\text { que objetos visuais } \\
\text { podem potencializar } \\
\text { as respostas motoras, } \\
\text { mesmo na ausência de } \\
\text { uma intenção de agir }\end{array}$ & $\begin{array}{l}18 \text { jovens adultos } \\
\text { (média idade } \\
21,5 \pm 3,7 \text { ), sendo } \\
16 \text { mulheres. }\end{array}$ & Psicologia & $\begin{array}{l}\text { Acessório } \\
\text { externo }\end{array}$ & $\begin{array}{l}\text { TRe = Pressionar o cilindro } \\
\text { do acessório externo } \\
\text { quando o fundo da imagem } \\
\text { for alterada para a cor } \\
\text { azul e pressionar o cubo } \\
\text { quando a cor for amarela, } \\
\text { sobrepostas à imagens de } \\
\text { diferentes objetos. }\end{array}$ & $\begin{array}{l}\text { Os dados mostraram uma } \\
\text { interação entre o tipo de } \\
\text { resposta (muscular) e o } \\
\text { tipo de objeto, implicando a } \\
\text { geração de um plano motor } \\
\text { imediatamente após a } \\
\text { apresentação do objeto. }\end{array}$ \\
\hline $\begin{array}{l}\text { Cavézian } \\
\text { et al. } \\
(2010)^{15}\end{array}$ & Cortex & $\begin{array}{c}\text { Avaliar a qualidade } \\
\text { da visão central } \\
\text { em pacientes com } \\
\text { hemianopia (visão) com } \\
\text { o efeito de dois testes: } \\
\text { tarefas de detecção } \\
\text { e categorização de } \\
\text { imagens. }\end{array}$ & $\begin{array}{l}36 \text { idosos (média } \\
\text { idade } 61,5 \pm 11,6) \\
\text { sendo } 11 \mathrm{com} \\
\text { lesão cerebral. }\end{array}$ & $\begin{array}{l}\text { Reabilitação } \\
\text { visual } \\
\text { (Oftalmologia) }\end{array}$ & $\begin{array}{l}\text { Acessório } \\
\text { externo }\end{array}$ & $\begin{array}{c}\text { TRe = Pressionar uma } \\
\text { tecla do SR-BOX (Serial } \\
\text { Response Box) para uma } \\
\text { imagem de autoestrada e } \\
\text { outro botão para imagem } \\
\text { de cidade. }\end{array}$ & $\begin{array}{l}\text { Resultados sugerem } \\
\text { que a visão central } \\
\text { não está intacta na } \\
\text { hemianopia. O lado da } \\
\text { lesão seletivamente afeta } \\
\text { os tempos de reação nas } \\
\text { tarefas de deteção e } \\
\text { categorização. }\end{array}$ \\
\hline $\begin{array}{l}\text { Finlayson; } \\
\text { King; } \\
\text { Blundell } \\
(2008)^{16}\end{array}$ & Appetite & $\begin{array}{c}\text { Investigar o efeito da } \\
\text { manipulação da fome } \\
\text { por meio da satisfação } \\
\text { induzida por comida } \\
\text { em medidas implícita } \\
\text { e explícita e entre o } \\
\text { processo de gostar e } \\
\text { querer. }\end{array}$ & $\begin{array}{l}70 \text { adultos (média } \\
\text { idade } 21,8 \pm 0,85 \text { ), } \\
\text { sendo } 30 \text { homens } \\
\text { e } 40 \text { mulheres. }\end{array}$ & $\begin{array}{l}\text { Controle } \\
\text { do apetite } \\
\text { (Segurança } \\
\text { Alimentar e } \\
\text { Nutricional) }\end{array}$ & Mouse & $\begin{array}{l}\text { TRe = Selecionar uma } \\
\text { opção com o clique do } \\
\text { mouse no aparecimento da } \\
\text { imagem de comida. }\end{array}$ & $\begin{array}{l}\text { Dados indicam que o } \\
\text { processo computadorizado } \\
\text { entre gostar e querer } \\
\text { apresenta dois distintos } \\
\text { processos psicológicos } \\
\text { que contribuem de forma } \\
\text { independente com a } \\
\text { preferência de alimentos, } \\
\text { com implicações para o } \\
\text { controle do apetite. }\end{array}$ \\
\hline
\end{tabular}


Tabela 2: Continuação

\begin{tabular}{|c|c|c|c|c|c|c|c|}
\hline Estudo & Periódico & Objetivo & Amostra & $\begin{array}{c}\text { Área de } \\
\text { aplicação e/ou } \\
\text { especialização }\end{array}$ & AR & $\begin{array}{l}\text { Tipo de TR e } \\
\text { descrição da } \\
\text { tarefa de TR }\end{array}$ & Resultados \\
\hline $\begin{array}{l}\text { Hartfield; } \\
\text { Conture } \\
(2006)^{9}\end{array}$ & $\begin{array}{l}\text { Journal of } \\
\text { Fluency } \\
\text { Disorders }\end{array}$ & $\begin{array}{c}\text { Tempo de reação na } \\
\text { fala de gagos e não } \\
\text { gagos. }\end{array}$ & $\begin{array}{c}26 \text { crianças (3 a } 7 \\
\text { anos), sendo } 13 \\
\text { gagos. }\end{array}$ & $\begin{array}{l}\text { Neurolinguagem } \\
\text { (Psicologia } \\
\text { Cogniftiva e da } \\
\text { Linguagem) }\end{array}$ & $\begin{array}{l}\text { Acessório } \\
\text { externo } \\
\text { (microfone) }\end{array}$ & $\begin{array}{c}\text { TR linguagem = Verbalizar } \\
\text { o nome da figura mostrada } \\
\text { o mais rapidamente } \\
\text { possível. }\end{array}$ & $\begin{array}{l}\text { Resultados sugerem } \\
\text { que os gagos tendem a } \\
\text { organizar a informação } \\
\text { lexical mais funcionalmente } \\
\text { do que fisicamente e } \\
\text { que esta tendência pode } \\
\text { estar relacionada com } \\
\text { dificuldades de estabelecer } \\
\text { fala e linguagem normal. }\end{array}$ \\
\hline $\begin{array}{l}\text { Wilson; } \\
\text { Maruff; } \\
\text { Lum } \\
(2003)^{17}\end{array}$ & $\begin{array}{l}\text { Human } \\
\text { Movement } \\
\text { Science }\end{array}$ & $\begin{array}{l}\text { Determinar se crianças } \\
\text { com desordem no } \\
\text { desenvolvimento } \\
\text { da coordenação } \\
\text { demonstram } \\
\text { procedimento de } \\
\text { aprendizagem normal. }\end{array}$ & $\begin{array}{l}20 \text { crianças (8 a } \\
12 \text { anos), sendo } 10 \\
\text { com distúrbio de } \\
\text { coordenação. }\end{array}$ & $\begin{array}{l}\text { Movimento } \\
\text { humano }\end{array}$ & Teclado & $\begin{array}{c}\text { TRs = Pressionar as teclas } \\
\text { "D", "F", "G" ou "H" conforme } \\
\text { a sequência apresentada } \\
\text { no monitor. }\end{array}$ & $\begin{array}{l}\text { Aprendizagem processual } \\
\text { para movimentos } \\
\text { simples sequenciais } \\
\text { parece estar intacta em } \\
\text { crianças com desordem } \\
\text { no desenvolvimento da } \\
\text { coordenação. }\end{array}$ \\
\hline $\begin{array}{l}\text { Ehrman } \\
\text { et al. } \\
(2002)^{13}\end{array}$ & $\begin{array}{c}\text { Drug and } \\
\text { Alcohol } \\
\text { Dependence }\end{array}$ & $\begin{array}{l}\text { Prever que ex-fumantes } \\
\text { apresentarão menor } \\
\text { viés para eventos } \\
\text { relacionados ao } \\
\text { tabagismo que os } \\
\text { fumantes. }\end{array}$ & $\begin{array}{l}30 \text { adultos (média } \\
\text { idade } 32,8 \pm 10,2 \text { ), } \\
\text { sendo } 7 \text { fumantes } \\
\text { e } 23 \text { não fumantes. }\end{array}$ & $\begin{array}{l}\text { Uso de drogas } \\
\text { (Toxicologia) }\end{array}$ & $\begin{array}{c}\text { Acessório } \\
\text { externo }\end{array}$ & $\begin{array}{l}\text { TRe = Pressionar o botão } \\
\text { esquerdo ou direito de } \\
\text { uma caixa com botões de } \\
\text { resposta que acompanha } \\
\text { o software em função de } \\
\text { imagens relacionadas ao } \\
\text { cigarro ou não. }\end{array}$ & $\begin{array}{l}\text { Os fumantes exibiram } \\
\text { significativamente maior } \\
\text { viés atencional para } \\
\text { estímulos relacionados } \\
\text { ao cigarro do que os não } \\
\text { fumantes. }\end{array}$ \\
\hline
\end{tabular}

AR: acessório utilizado para resposta ao estímulo na tarefa de tempo de reação; TR: tempo de reação simples; TRe: tempo de reação de escolha

publicou dois artigos; as demais pesquisas foram publicadas em distintos periódicos.

Em relação ao número de participantes, as pesquisas com adultos variaram entre 18 e 139 sujeitos (média de 57,6). As pesquisas com crianças foram realizadas com problemas de gagueira e com distúrbios de coordenação. A maior parte das pesquisas utilizou um acessório externo para registrar a resposta. Apenas uma pesquisa utilizou-se do mouse para registro da resposta. Todas as pesquisas propuseram tarefas de TR distintas.

Os trabalhos de Hartfield e Conture ${ }^{9}$ utilizaram o software E-Prime ${ }^{\circledR}$ para avaliar o desempenho no TR de forma direta para investigar a influência do feedback na motivação em adultos saudáveis, enquanto Eckner, Chandran e Richardson ${ }^{10}$ e Forestell et al. ${ }^{11}$, na fala de crianças gagas e não gagas. Os demais artigos utilizaram a tarefa de TR para avaliar outra variável, como: (a) indicadores de dependência em adolescentes $^{12}$ ou adultos ${ }^{13}$; (b) planos motores em jovens adultos ${ }^{14}$; (c) qualidade da visão em idosos ${ }^{15}$; (d) apetite em adultos ${ }^{11,16}$; (e) desenvolvimento da coordenação em crianças $^{17}$; (f) distúrbios da atenção em pacientes com doença de Parkinson ${ }^{18}$.

As tarefas propostas são todas distintas, havendo uma predominância no uso de imagens. Somente os estudos de van Holst et al. ${ }^{12}$ e Ehrman et al. ${ }^{13}$ se assemelham na execução da tarefa - ambos mostram duas imagens, uma de cada lado do monitor, que desaparecem em seguida para que o participante selecione o local correto do aparecimento de um indicador em um dos lados. Para Ehrman et al. ${ }^{13}$, que estudaram fumantes, as figuras se referiam a imagens relacionadas ao tabagismo. Para Van Holst et al..$^{12}$, que estudaram jogadores de videogame, as imagens continham temas de jogos de videogame. O primeiro utilizou um acessório externo e o segundo, o teclado. As tarefas de avaliação das tendências em comportamento alimentar utilizaram figuras de comidas com ou sem alto valor calórico em situações alternadas ${ }^{11,16}$.

De modo geral, o TR é classificado como simples e de escolha. O TR simples foi utilizado no estudo de Eckner, Chandran e Richardson ${ }^{10}$; Dujardin et al. ${ }^{18}$ utilizaram tanto tarefas de TR simples quanto de escolha. Tarefas de TR simples requerem somente o reconhecimento do estímulo e do movimento e são curtas na duração. $\mathrm{O}$ TR de escolha predominou nos estudos $(\mathrm{n}=5)$. Tarefas com TR mais complexas requerem funções cognitivas adicionais, como escolha e discriminação entre estímulo ${ }^{19}$.

\section{DISCUSSÃO}

O tempo de reação (TR) é um tema bastante discutido principalmente na Psicologia moderna ${ }^{20}$. Na Psicologia do Esporte, um dos estudos pioneiros envolvendo o esporte utilizou uma tarefa de $\mathrm{TR}^{21}$.

Para desenvolver um estudo com tarefas de TR com uso do computador, os pesquisadores podem aprender uma linguagem de programação e desenvolver seu próprio software ou usar um dos vários pacotes de software disponíveis para a criação das tarefas e executar suas pesquisas ${ }^{4}$.

Alguns autores destacam que a falta de padronização nos testes, tanto em relação à tarefa quanto ao estímulo, dificulta uma análise mais refinada das tendências do $\mathrm{TR}^{22-25}$.

$\mathrm{O}$ que podemos observar nos resultados da pesquisa aqui apresentada é que essa dificuldade ocorre mesmo quando a pesquisa se utiliza do mesmo software para geração e controle da tarefa de TR. O software E-Prime ${ }^{\circledR}$ (versão 2004) foi descrito por Stahl $^{4}$ como sendo um pacote de software composto por cinco módulos, nos quais os experimentos são criados com E-Studio e a execução com ERun, e três ferramentas adicionais estão disponíveis para mesclar e analisar os dados além de recuperar dados 
perdidos. O registro das respostas é realizado por meio do teclado, mouse ou caixas de resposta externas ligadas à porta serial.

Existe a preocupação de diversos autores com relação à precisão da medida de tempo utilizando o computador com sistema operacional multitarefa como o Windows, em que a execução de outro programa pode interferir no desempenho do registro da fração de milissegundos da execução de uma tarefa de $\mathrm{TR}^{4,26-28}$. Além disso, a qualidade dos acessórios empregados para resposta também apresenta diferenças significativas ${ }^{29}$.

Nesta pesquisa, observa-se maior uso do acessório externo fornecido com o pacote de software ${ }^{9,13-15,18,30}$, mas o teclado veio em segundo lugar ${ }^{10-12,17}$. Somente o estudo de Finlayson, King e Blundell ${ }^{16}$ utilizou um mouse para registro das respostas. Nenhum dos autores mencionou qualquer tipo de preocupação com o acessório utilizado para a resposta.

No estudo de Dujardin et al..$^{18}$, o TR foi aplicado em pacientes Parkinsonianos com os resultados evidenciando déficits nos componentes executivos da atenção. Além de um TR mais lento e com maior variabilidade, apresentaram um desempenho comprometido (no tempo de resposta e na precisão) em situações que requerem flexibilidade mental, apresentando baixa capacidade de focar a atenção, resistir à interferência e dividir recursos de atenção.

A seguir são apresentados os demais estudos revisados, separando-os por tema.

\section{O efeito da motivação sobre 0 desempenho no tempo de reação}

Eckner, Chandran e Richardson ${ }^{10}$ investigaram a influência do feedback e da motivação no desempenho durante 2 testes de tempo de reação simples visual em 31 adultos saudáveis (com média de idade de 54£15) que estavam na sala de espera de uma clínica de reabilitação aguardando pacientes em tratamento. Os autores afirmaram que os participantes motivados são mais propensos a apresentar um nível elevado de esforço durante a tarefa de TR do que os participantes desmotivados ou entediados.

Evidências sugerem que a atenção e o controle da motivação ou da orientação para a tarefa podem melhorar a eficácia com motivação intrínseca (como o interesse do participante na tarefa) e extrínseca por meio de incentivos externos (como pontos ou recompensas em dinheiro) $)^{31}$.

Motivadores extrínsecos podem servir como uma fonte imediata de feedback durante a execução da tarefa, informando os participantes de seus níveis atuais e anteriores de desempenho, e levando-os a ajustar a sua abordagem estratégica se os resultados são mais baixos do que o desejado ${ }^{31}$.

O estudo de Eckner, Chandran e Richardson ${ }^{10}$ comparou o desempenho na execução da mesma tarefa usando o E-Prime ${ }^{\circledR}$, e em uma delas o resultado do desempenho era mostrado após a execução de cada tentativa.

Van Holst et al. ${ }^{12}$ utilizaram feedback apenas durante a fase de familiarização dos participantes com a tarefa. Os demais estudos selecionados nesta pesquisa não utilizaram feedback com os participantes.

\section{Transtornos relacionados ao uso de substâncias e tempo de reação}

Van Holst et al. ${ }^{12}$ utilizaram a tarefa de TR para examinar, em 92 adolescentes do sexo masculino (com média de idade de 15,1 $\pm 1,27$ ) que dedicam a maior parte do seu tempo em jogos de computador ou videogame, se estes comportamentos estão relacionados com problemas de dependência. Os resultados sugerem a existência de um viés de atenção desses adolescentes semelhante aos dependentes de substâncias em relação aos mecanismos cognitivos emocionais.

Ehrman et al. ${ }^{13}$ analisaram 30 adultos (com média de idade de $32,8 \pm 10,2$ ), sendo 7 fumantes e 23 não fumantes, para prever se ex-fumantes apresentarão menor viés para eventos relacionados ao tabagismo do que os fumantes, encontrando que os fumantes exibiram significativamente maior viés atencional para estímulos relacionados ao cigarro do que os não fumantes.

\section{Tempo de reação na identificação de tendências no comportamento alimentar}

Finlayson, King e Blundell ${ }^{16}$ utilizaram a tarefa de TR para investigar o efeito da manipulação da fome por meio da satisfação induzida por comida em medidas implícita e explícita e entre o processo de gostar e querer em 70 adultos (com média de idade de $21,8 \pm 0,85)$. Os resultados indicaram que o processo computadorizado entre gostar e querer apresenta dois distintos processos psicológicos que contribuem de forma independente com a preferência de alimentos, com implicações para o controle do apetite.

Havermans et al. ${ }^{32}$ afirmam que as mulheres com sobrepeso ou obesas ainda tendem a comer alimentos ricos em calorias, apesar de demonstrarem uma intenção clara para evitar esses alimentos na execução de tarefas de TR, e que talvez os excessos só ocorram em situações em que a pessoa simplesmente não pode evitar a exposição aos alimentos que apreciam.

Estes estudos utilizaram figuras de comida, exigindo uma escolha entre dois estímulos alimentares alvo, combinando com perguntas do tipo "Quão prazeroso seria experimentar um pouco deste alimento agora?" e "Quanto você quer um pouco deste alimento agora?".

Para determinar como as mulheres que têm hábitos alimentares distintos (comem desenfreadamente ou de forma contida) demonstram atenção seletiva para imagens de comida com alto teor calórico, Forestell et al. ${ }^{11}$ aplicaram dois testes: quando as mulheres estavam saciadas, os alimentos de alto teor calórico tinham pouco efeito sobre o desempenho, independentemente de seus hábitos alimentares; quando estavam com fome, os resultados forneceram evidência de que os alimentos de alto teor calórico induziram respostas diferentes nas mulheres de hábitos contidos daquelas sem restrições.

O uso de tarefas de TR parece ser uma medida sensível para identificar tendências no comportamento alimentar.

\section{O tempo de reação e transtornos da visão}

Os testes com tarefas de TR têm se mostrado eficazes em análises da visão principalmente na reabilitação visual ${ }^{33,34}$. 
Nesta revisão, Cavézian et al. ${ }^{15}$ avaliaram a qualidade da visão central em pacientes com hemianopia após lesão cerebral em 11 idosos comparando com 25 idosos sem lesão (com média geral de idade de $61,5 \pm 11,6)$. Os resultados sugerem que a visão central não está intacta na hemianopia e que o lado da lesão seletivamente afeta os tempos de reação nas tarefas de detecção e categorização.

\section{Tempo de reação e comportamento motor}

Wilson, Maruff e Lum ${ }^{17}$ propuseram uma tarefa de TR para determinar se crianças com desordem no desenvolvimento da coordenação demonstram procedimento de aprendizagem normal. O estudo foi realizado com 10 crianças com distúrbio da coordenação e com 10 crianças que não apresentavam nenhum distúrbio. Os resultados indicaram que a aprendizagem processual para movimentos simples sequenciais parece estar intacta em crianças com desordem no desenvolvimento da coordenação, sugerindo uma forte implicação da sequência de movimentos simples com os circuitos córtico-estriatais.

O estudo de Makris, Hadar e Yarrow ${ }^{14}$ analisou a evolução temporal de planos motores oferecida por objetos comuns para verificar a teoria de affordances que implica que objetos visuais podem potencializar as respostas motoras, mesmo na ausência de uma intenção de agir. O estudo foi realizado com 18 jovens adultos, com média de idade de 21,5 $\pm 3,7$ anos, afirmando que os dados mostraram uma interação entre o tipo de resposta (muscular) e o tipo de objeto, implicando a geração de um plano motor imediatamente após a apresentação do objeto. Esses resultados proporcionam um apoio adicional à noção de que as propriedades físicas dos objetos ativam, automaticamente, códigos motores específicos, mas também demonstram que essa influência é relativamente rápida e de curta duração. Resultados semelhantes são reportados por Suzuki, Takagi e Sugawara ${ }^{35}$ e Ranganathan et al. ${ }^{36}$.

\section{Tempo de reação para avaliação de transtornos da linguagem}

A comparação de resultados encontrados em tarefas de TR com gagos e não gagos demonstra tendências importantes e merece uma investigação mais aprofundada ${ }^{37}$, principalmente porque a extensão de déficits não linguísticos e suas relações com os déficits de linguagem ainda não são suficientemente claras ${ }^{38,39}$.

O estudo de Hartfield e Conture ${ }^{9}$ avaliou o TR na fala de 13 crianças com gagueira e 13 crianças não gagas, e os resultados sugerem que os gagos tendem a organizar a informação lexical mais funcionalmente do que fisicamente e que esta tendência pode estar relacionada com dificuldades de estabelecer fala e linguagem normal.

Conture participou de outro estudo com gagos e não gagos (com 12 meninos em cada grupo $)^{30}$, no qual os autores apresentam resultados que são inconsistentes com resultados de outros estudos empíricos que investigam a relação entre atenção, emoção e gagueira infantil, afirmando que os meninos gagos em idade pré-escolar não são menos capazes de regular sua atenção do que os não gagos. Os autores concluem que a atenção e sua regulação são associadas com a gagueira, mas a natureza dessa associação ainda não é clara e as circunstâncias dela continuam a ser mal-entendidas, enfatizando a importância de se aplicar a mesma metodologia para que os resultados possam ser comparados.

\section{CONCLUSÃO}

A presente pesquisa analisou as publicações sobre o uso do software E-Prime ${ }^{\mathbb{B}}$ para execução de tarefas de TR na base de dados ScienceDirect.

A análise demonstrou que as publicações sobre o TR vêm sendo realizadas com diferentes temas de pesquisa e que as tarefas propostas são bastante distintas, mas com predominância no uso de imagens. Alguns estudos se utilizaram de um acessório externo, sendo o teclado o segundo recurso mais utilizado. Somente um estudo fez uso de um mouse para registro da resposta.

Identificou-se o uso de tarefas de TR para avaliar o efeito da motivação sobre o desempenho no TR, para análise dos transtornos relacionados ao uso de substâncias, no comportamento alimentar, em transtornos da visão, no comportamento motor e em transtornos da linguagem, além de auxiliar na investigação dos distúrbios da atenção em pacientes parkinsonianos.

TambémficaclaraaaplicabilidadedeestudoscomtarefasdeTR,não havendo uma predominância nos temas estudados, mas percebe-se uma forte relação entre TR e medidas de saúde física e cognitiva.

\section{REFERÊNCIAS}

1. Cinaz B, Vogt C, Arnrich B, Troster G. A wearable user interface for measuring reaction time. In: Keyson DV, Maher ML, Streitz $\mathrm{N}$, Cheok A, Augusto JC, Wichert R, et al. Ambient intelligence. Berlim: Springer; 2011. p. 41-50. http://dx.doi.org/10.1007/978-3-642-25167-2_5

2. Cinaz B, Vogt C, Arnrich B, Troster G. Implementation and evaluation of wearable reaction time tests. Pervasive Mobile Computing. 2012;8(6):813-21. http://dx.doi.org/10.1016/j.pmcj.2012.06.006

3. Erickson GB, Citek K, Cove M, Wilczek J, Linster C, Bjarnason B, et al. Reliability of a computer-based system for measuring visual performance skills. Optometry. 2011;82(9):528-42. http://dx.doi.org/10.1016/j.optm.2011.01.012
4. Stahl C. Software for generating psychological experiments. Exp Psychol. 2006;53(3):218-32.

http://dx.doi.org/10.1027/1618-3169.53.3.218

5. Wild K, Howieson D, Webbe F, Seelye A, Kaye J. Status of computerized cognitive testing in aging: a systematic review. Alzheimers Dement. 2008;4(6):428-37.

http://dx.doi.org/10.1016/j.jalz.2008.07.003

6. Ozyemisci-Taskiran O, Gunendi Z, Bolukbasi N, Beyazova M. The effect of a single session submaximal aerobic exercise on premotor fraction of reaction time: an electromyographic study. Clin Biomech. 2008;23(2):231-5 http://dx.doi.org/10.1016/j.clinbiomech.2007.08.027

7. Liberati A, Altman DG, Tetzlaff J, Mulrow C, Gotzsche PC Ioannidis JPA, et al. The prisma statement for reporting systematic 
reviews and meta-analyses of studies that evaluate healthcare interventions: explanation and elaboration. BMJ. 2009;339:b2700. http://dx.doi.org/10.1136/bmj.b2700

8. Sampaio RF, Mancini M. Estudos de revisão sistemática: um guia para síntese criteriosa da evidência científica. Rev Bras Fisioter. 2007;11(1):83-9.

http://dx.doi.org/10.1590/S1413-35552007000100013

9. Hartfield KN, Conture EG. Effects of perceptual and conceptual similarity in lexical priming of young children who stutter: preliminary findings. J Fluency Disord. 2006;31(4):303-24. http://dx.doi.org/10.1016/j.jfludis.2006.08.002

10. Eckner JT, Chandran S, Richardson JK. Investigating the role of feedback and motivation in clinical reaction time assessment. PMR. 2011;3(12):1092-97

http://dx.doi.org/10.1016/j.pmrj.2011.04.022

11. Forestell CA, Lau P, Gyurovski II, Dickter CL, Haque SS. Attentional biases to foods: the effects of caloric content and cognitive restraint. Appetite. 2012;59(3):748-54. http://dx.doi.org/10.1016/j.appet.2012.07.006

12. van Holst RJ, Lemmens JS, Valkenburg PM, Peter J, Veltman DJ, Goudriaan AE. Attentional bias and disinhibition toward gaming cues are related to problem gaming in male adolescents. J Adolesc Health. 2012;50(6):541-6.

http://dx.doi.org/10.1016/j.jadohealth.2011.07.006

13. Ehrman RN, Robbins SJ, Bromwell MA, Lankford ME, Monterosso JR, O'Brien CP. Comparing attentional bias to smoking cues in current smokers, former smokers, and non-smokers using a dot-probe task. Drug Alcohol Depend. 2002;67(2):185-91. http://dx.doi.org/10.1016/S0376-8716(02)00065-0

14. Makris S, Hadar AA, Yarrow K. Viewing objects and planning actions: on the potentiation of grasping behaviours by visual objects. Brain Cogn. 2011;77(2):257-64. http://dx.doi.org/10.1016/j.bandc.2011.08.002

15. Cavézian C, Gaudry I, Perez C, Coubard O, Doucet G, Peyrin C, et al. Specific impairments in visual processing following lesion side in hemianopic patients. Cortex. 2010;46(9):1123-31. http://dx.doi.org/10.1016/j.cortex.2009.08.013

16. Finlayson $G$, King N, Blundell J. The role of implicit wanting in relation to explicit liking and wanting for food: implications for appetite control. Appetite. 2008;50(1):120-7. http://dx.doi.org/10.1016/j.appet.2007.06.007

17. Wilson $\mathrm{PH}$, Maruff $\mathrm{P}$, Lum J. Procedural learning in children with developmental coordination disorder. Hum Mov Sci. 2003;22(4-5):515-26. http://dx.doi.org/10.1016/j.humov.2003.09.007

18. Dujardin K, Tard C, Duhamel A, Delval A, Moreau C, Devos D, et al. The pattern of attentional deficits in parkinson's disease. Parkinsonism Relat Disord. 2013;19(3):300-5.

http://dx.doi.org/10.1016/j.parkreldis.2012.11.001

19. Kelly DD, Murphy BA, Backhouse DP. Use of a mental rotation reaction time paradigm to measure the effects of upper cervical adjustments on cortical processing: a pilot study. J Manipulative Physiol Ther. 2000;23(4):246-51. http://dx.doi.org/10.1067/mmt.2000.106099

20. Zajdel R, Nowak D. Simple and complex reaction time measurement a preliminary evaluation of new approach and diagnostic tool. Comput Biol Med. 2007;37(12):1724-30. http://dx.doi.org/10.1016/j.compbiomed.2007.04.008

21. Kornspan AS. Applied research E.W. Scripture and the Yale Psychology Laboratory: studies related to athletes and physical activity. Sport Psychol. 2007;21(2):152-69.

22. Silverman IW. Sex differences in simple visual reaction time: a historical meta-analysis. Sex Roles. 2006;54(1-2):57-8. http://dx.doi.org/10.1007/s11199-006-8869-6
23. Silverman IW. Simple reaction time: it is not what it used to be. Am J Psychol. 2010;123(1):39-50.

24. Deary IJ, Liewald D, Nissan J. A free, easy-to-use, computer-based simple and four-choice reaction time programme: the deary-liewald reaction time task. Behav Res Methods. 2011;43(1):258-68. http://dx.doi.org/10.3758/s13428-010-0024-1

25. Li X, Liang Z, Kleiner M, Lu Z-L. Rtbox: a device for highly accurate response time measurements. Behav Res Methods. 2010;42(1):212-25. http://dx.doi.org/10.3758/BRM.42.1.212

26. Ohyanagi T, Sengoku Y. A solution for measuring accurate reaction time to visual stimuli realized with a programmable microcontroller. Behav Res Methods. 2010;42(1):242-53. http://dx.doi.org/10.3758/BRM.42.1.242

27. Neath I, Earle A, Hallett D, Surprenant AM. Response time accuracy in apple macintosh computers. Behav Res Methods. 2011;43(2):353-62. http://dx.doi.org/10.3758/s13428-011-0069-9

28. Spruyt A, Clarysse J, Vansteenwegen D, Baeyens F, Hermans D. Affect 4.0 a free software package for implementing psychological and psychophysiological experiments. Exp Psychol. 2010;57(1):36-5. http://dx.doi.org/10.1027/1618-3169/a000005

29. Cernich AN, Brennana DM, Barker LM, Bleiberg J. Sources of error in computerized neuropsychological assessment. Arch Clin Neuropsychol. 2007;22(Suppl 1):39-48. http://dx.doi.org/10.1016/j.acn.2006.10.004

30. Johnson KN, Conture EG, Walden TA. Efficacy of attention regulation in preschool-age children who stutter: a preliminary investigation. J Commun Disord. 2012;45(4):263-78. http://dx.doi.org/10.1016/j.jcomdis.2012.04.001

31. Garrett DD, MacDonald SWS, Craik FIM. Intraindividual reaction time variability is malleable: feedback- and education-related reductions in variability with age. Front Hum Neurosci. 2012;6:101. http://dx.doi.org/10.3389/fnhum.2012.00101

32. Havermans RC, Giesen J, Houben K, Jansen A. Weight, gender, and snack appeal. Eat Behav. 2011;12(2):126-30. http://dx.doi.org/10.1016/j.eatbeh.2011.01.010

33. Poggel DA, Treutwein B, Strasburger $H$. Time will tell: deficits of temporal information processing in patients with visual field loss. Brain Res. 2011;1368:196-207 http://dx.doi.org/10.1016/j.brainres.2010.10.065

34. Wolffsohn JS, Bhogal G, Shah S. Effect of uncorrected astigmatism on vision. J Cataract Refract Surg. 2011;37(3):454-60. http://dx.doi.org/10.1016/j.jcrs.2010.09.022

35. Suzuki T, Takagi M, Sugawara K. Affordance effects in grasping actions for graspable objects: electromyographic reaction time study. Percept Mot Skills. 2012;115(3):881-90. http://dx.doi.org/10.2466/26.22.24.PMS.115.6.881-890

36. Ranganathan R, Lee MH, Brown AJ, Newell KM. Grasping possibilities for action: influence of object function and action capabilities. Hum Mov Sci. 2011;30(6):1102-14 http://dx.doi.org/10.1016/j.humov.2010.11.012

37. Smits-Bandstra S. Methodological considerations in the measurement of reaction time in persons who stutter. J Fluency Disord. 2010;35(1):19-32. http://dx.doi.org/10.1016/j.jfludis.2009.12.002

38. Gabriel A, Maillart C, Stefaniak N, Lejeune C, Desmottes L, Meulemans T. Procedural learning in specific language impairment: effects of sequence complexity. J Int Neuropsychol Soc. 2013;19(3):264-71. http://dx.doi.org/10.1017/S1355617712001270

39. Moher D, Liberati A, Tetzlaff J, Altman DG; Prisma Group. Preferred reporting items for systematic reviews and meta-analyses: the Prisma statement. Ann Intern Med. 2009;151(4):264-9. http://dx.doi.org/10.7326/0003-4819-151-4-200908180-00135 